\title{
Coordinated and Integrated Leadership in Trauma System: A Forgotten Necessity in Iran
}

\author{
Vafa Rahimi Movaghar*
}

Department of Neurosurgery, Sina Trauma and Surgery Research Center, Tehran University of Medical Sciences, Tehran, Iran

\section{ABSTRACT}

Introduction: Trauma has the greatest burden of diseases in Iran. Most victims are young men. Road traffic crashes are the most common cause of trauma in Iran. The study has been designed to determine the most ignored need in trauma system in Iran. Materials and Methods: In two studies, the first one conducted by the Sina Trauma and Surgery Research Center (13 sessions) and the second one conducted by the section of Emergency department of Ministry of Health and Medical Education ( 8 sessions), trauma experts and policy makers discussed and reviewed trauma system strengths and weaknesses in the areas of prevention, treatment, rehabilitation, and surveillance of injured patients. Results: All organizations, including the Ministry of Health, office of injury prevention, emergency section, hospital and health care, Red Crescent, forensic medicine, police, ministry of interior, ministry of road, automobile manufacturers, municipality, media and journalists, NGOs, parliament, and judiciary all have faults and strengths. There were multiple and significant improvements for each organization. The most important defect was lack of unit leadership in the organized management of trauma that is responsible and answerable. In other word, there is lack of coordination and integration in this trauma system. Integrated monitoring system must also be permanent and included sufficient resources. Priority of teamwork in related agencies over individual work is necessary. Conclusion: For serious success in the prevention, treatment, rehabilitation, and surveillance of trauma system, we need a person or organization that is responsible for organizing, coordinating, and integrating efforts in the field of trauma.

\section{Key words:}

1. Leadership

2. Trauma, Nervous System

3. Organization and Administration

4. Delivery of Health Care, Integrated

* Corresponding Author: Vafa Rahimi Movaghar

E-mail:v_rahimi@sina.tums.ac.ir,v_rahimi@yahoo.com 


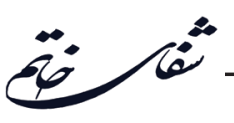

\section{رهبرى هماهنَ و يكيار جه در سيستهم تروما: يك ضرورت فراموش شده در ايران}

\section{وفا رحيمى موقر"}

كروه جراحى مغز و اعصاب، مركز تحقيقات تروما و جراحى سينا، دانشعاه علوم بزشكى تهران، تهران، ايران

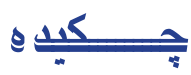

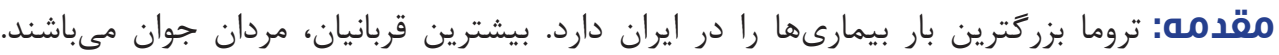

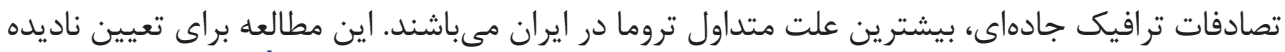

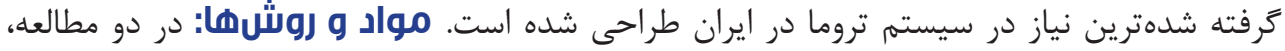

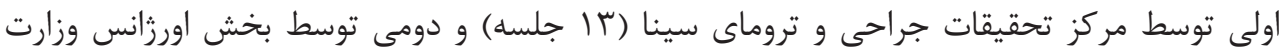

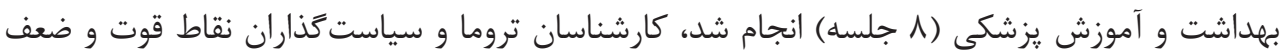

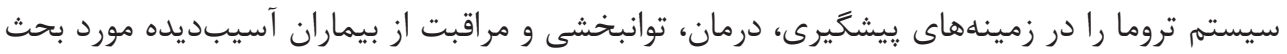

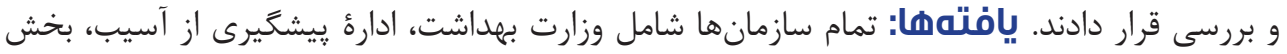

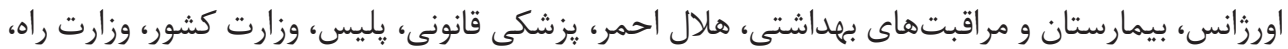
توليدكنند

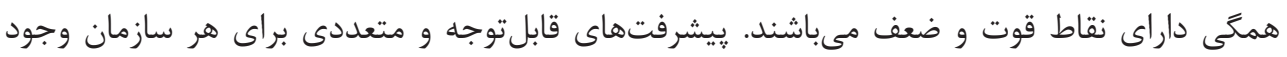

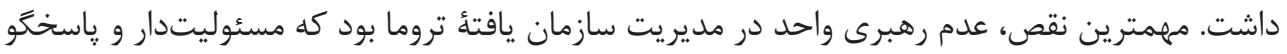

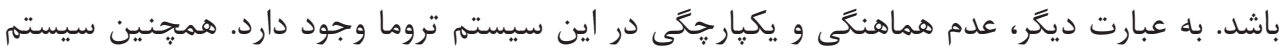

كليد وازهها:

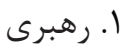

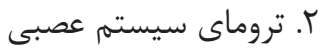

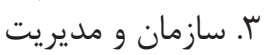

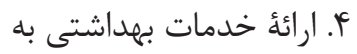
صورت يكيارجه

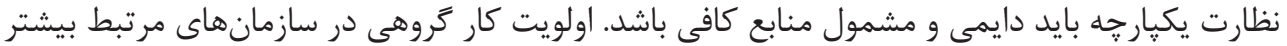

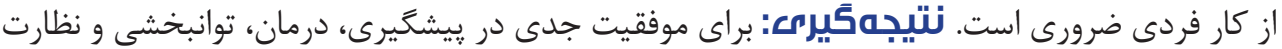

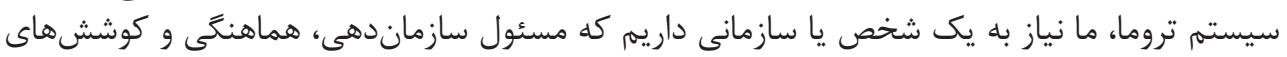
يكيار خهسازى در زمينأ تروما باشد.

" نويسنده مسئول: وفا رحيمى موقر آدرس الكترونيكى: v_rahimi@sina.tums.ac.ir,v_rahimi@yahoo.com 


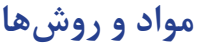

در مطالعهاى كه زير نظر مركز تحقيقات تروما و جراحى سينا

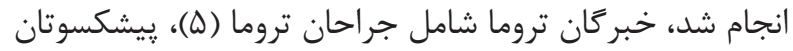

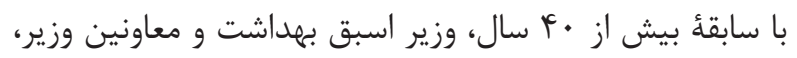

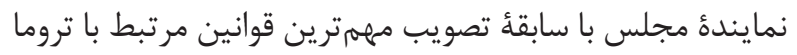

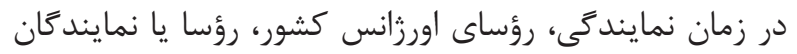

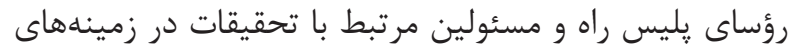

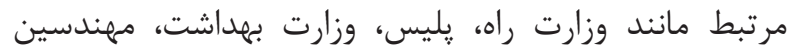

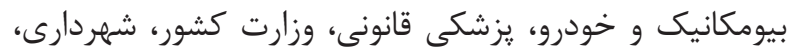

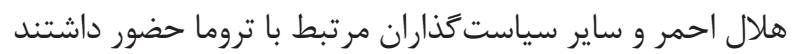

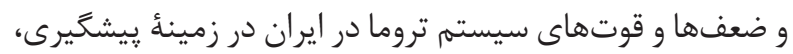

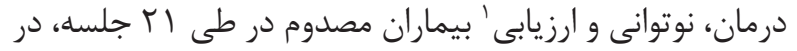

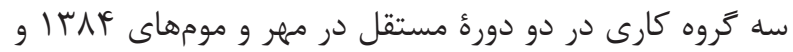

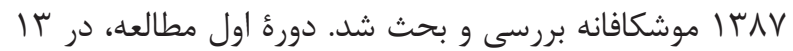

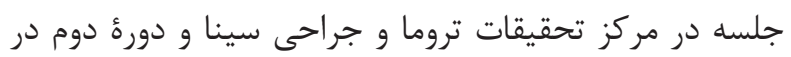

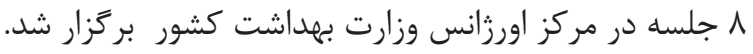

بافتهها

همة سازمانها شامل وزارت بهداشت، ادارئ بِيشخيرى از

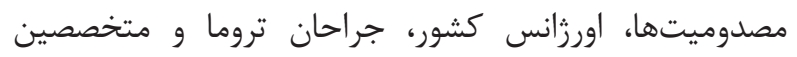

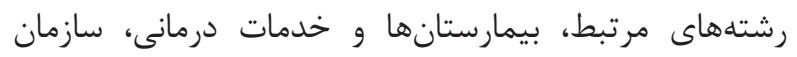

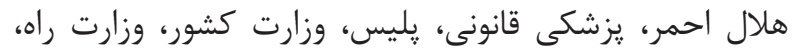

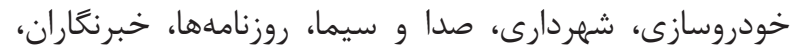

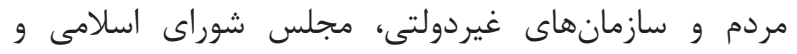

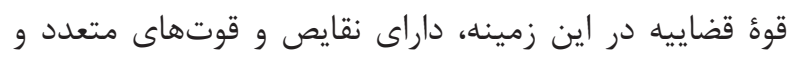

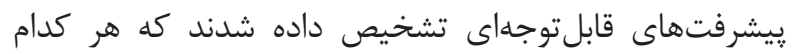

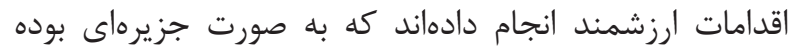

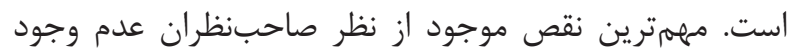

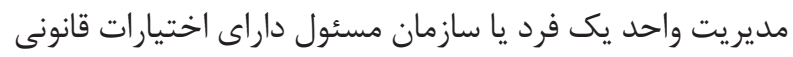

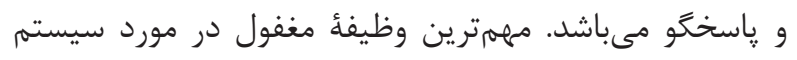

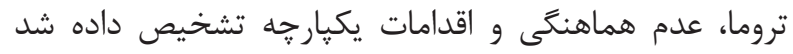

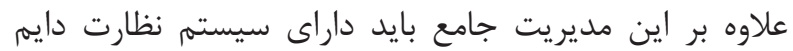

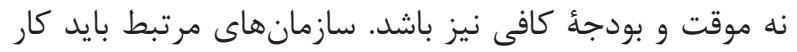

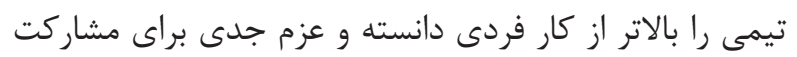
داشته و همكى نقديذير باشند.

بحث و نتيجه

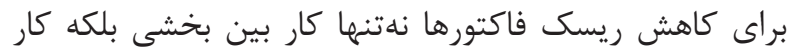

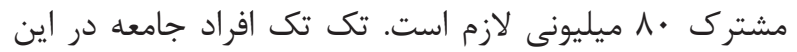

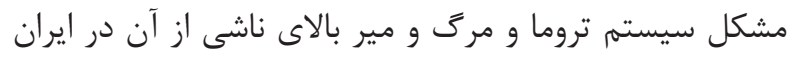

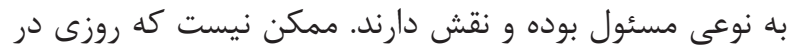

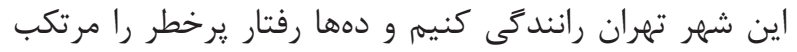

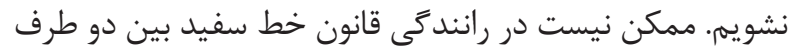

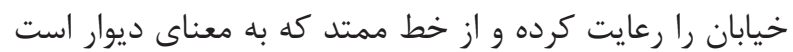

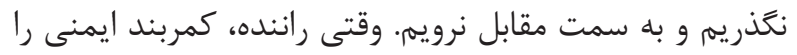

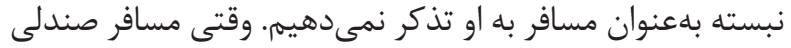

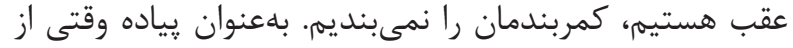

${ }^{1}$ Surveillance

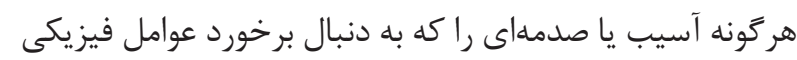

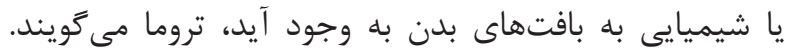

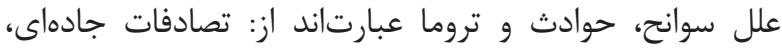

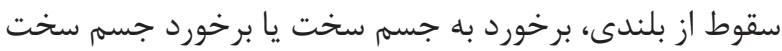

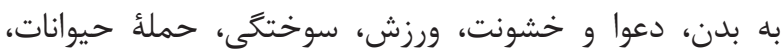

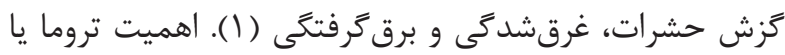

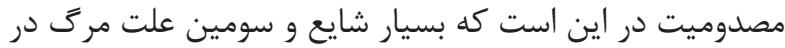

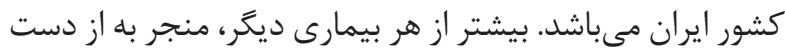

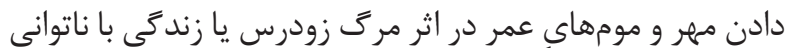

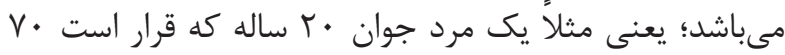

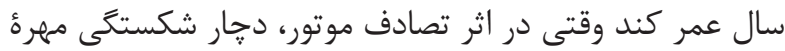

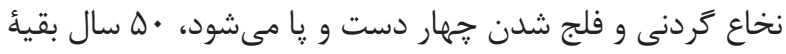

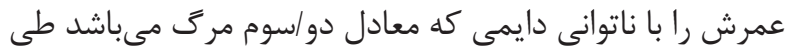

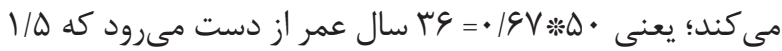

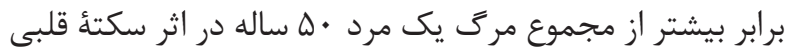
و مرد •9 ساله در اثر سرطان مى مباشد.

مردان جوان بيشتر دجار مصدوميت، تروما يا سوانح مىشوند

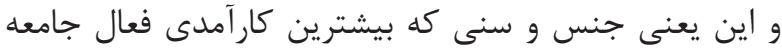

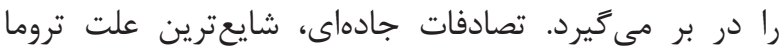

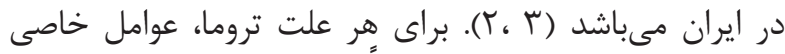

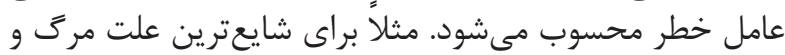

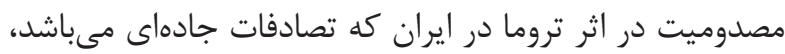

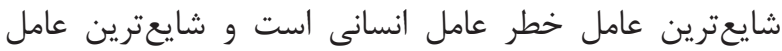

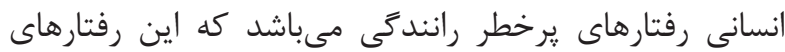

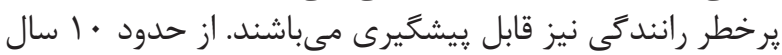

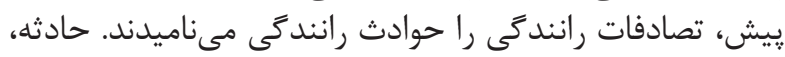

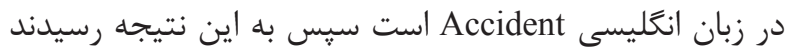

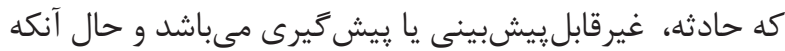

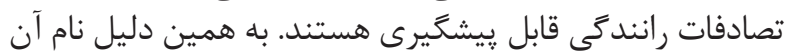

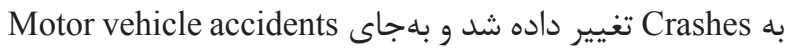
از Road traffic crashes استفاده شد.

رفتارهاى يرخطر رانندگى، فراوان مى باشند. مانند عبور اتومبيل

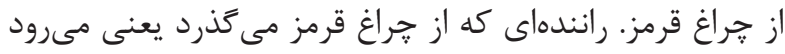

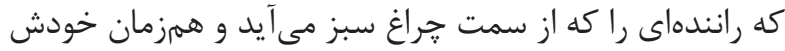

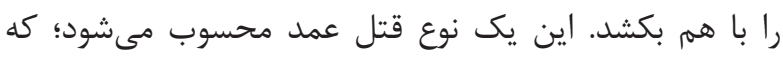

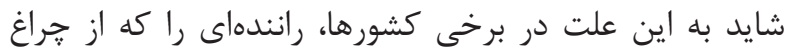

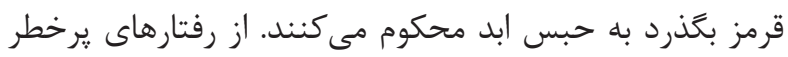

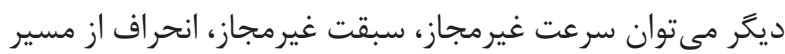

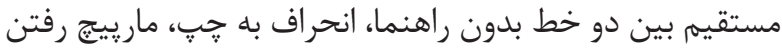

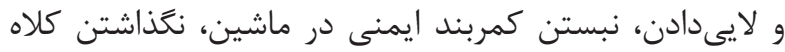

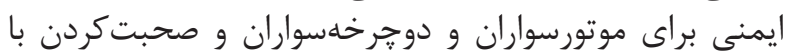

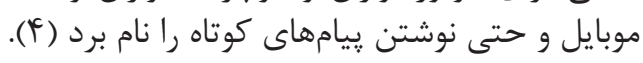

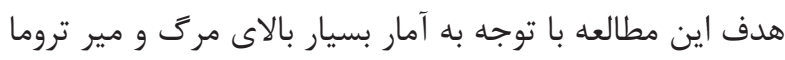

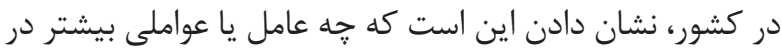
سيستم تروماى كشور مغفول مانده است. 
مترى، ميىايستند تا من علاوه بر آرامش با ايمنى از خيابان

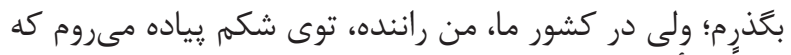

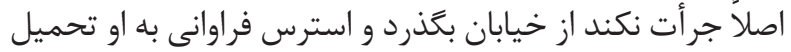

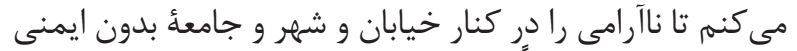

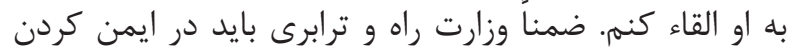

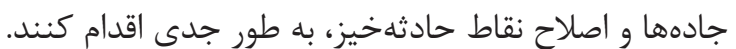

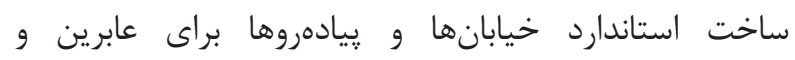

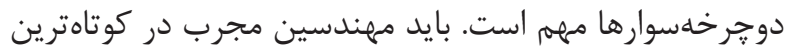

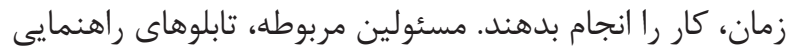

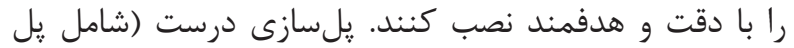

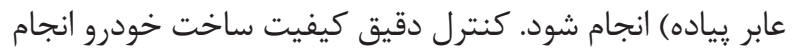

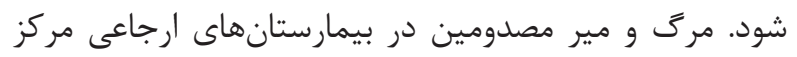

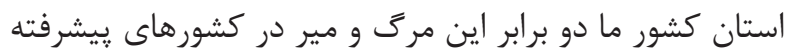

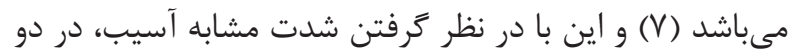

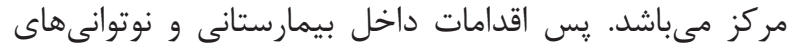

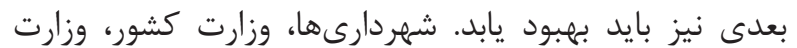

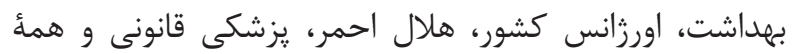

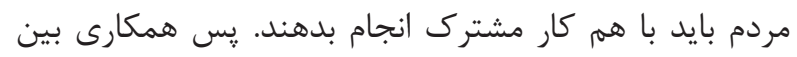

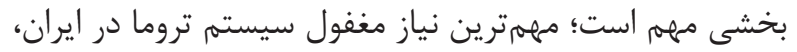

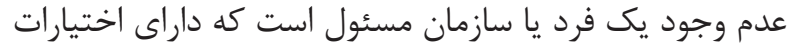

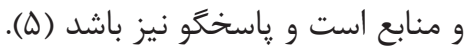

در مطالعات متعدد نقش عامل انسانى، شايعترين مورد در

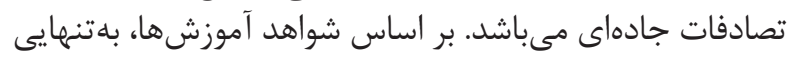

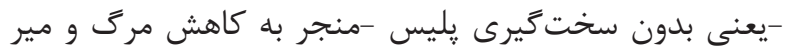

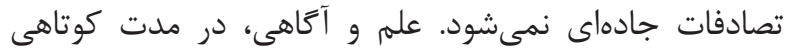

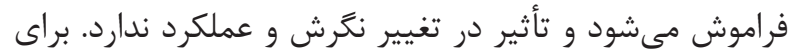

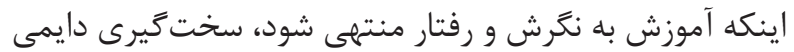

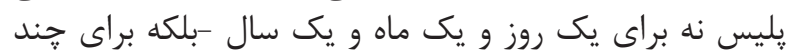

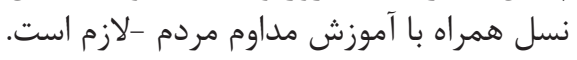

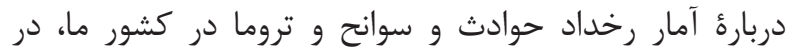

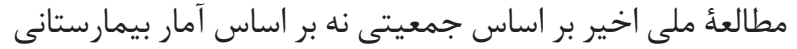

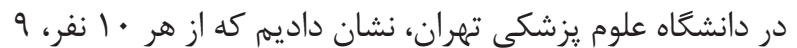

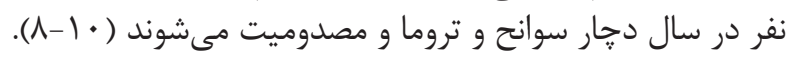

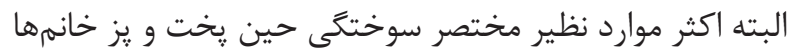

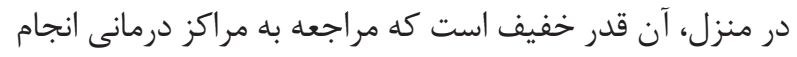

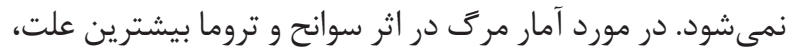

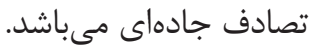

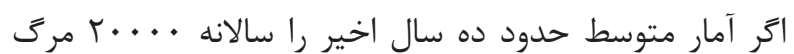

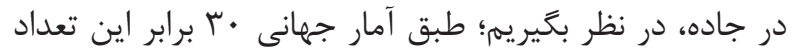

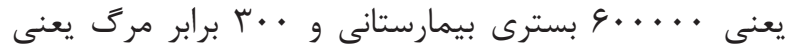

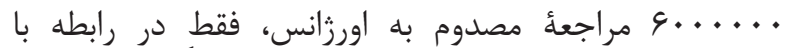

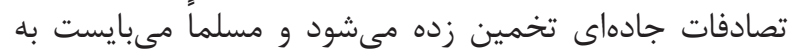

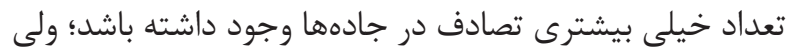

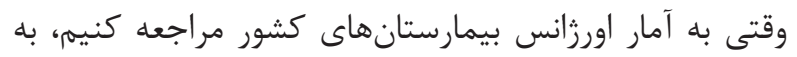

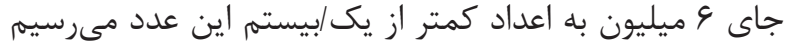
و اين كم شمارى ناشى از اشكال و عدم وجود ثبت دقيت ائ عديق موارد
اتوبوسهاى تندروى بزر گراه نواب يِياده مىشويم به جاى استفاده

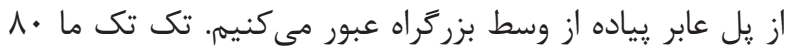

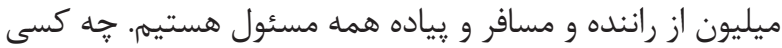

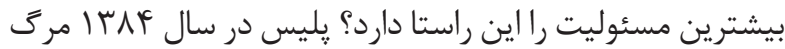

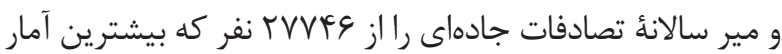

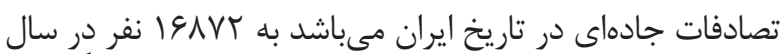

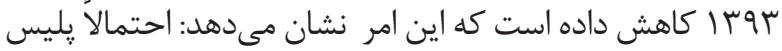

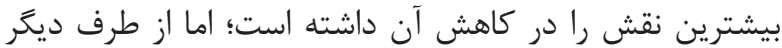

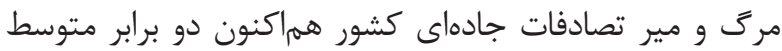

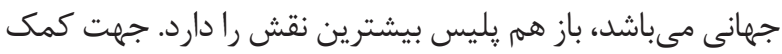

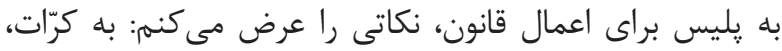

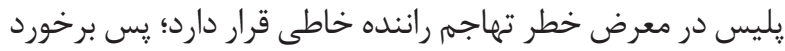

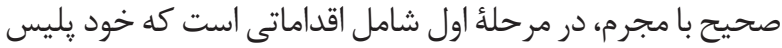
در معرض خطر قرار نخَيرد.

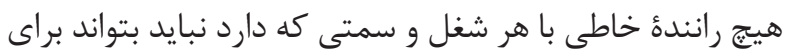

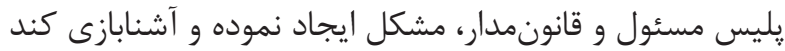

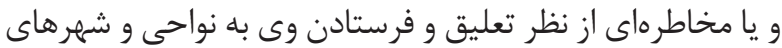

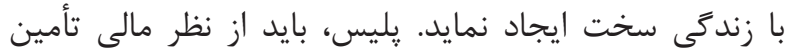

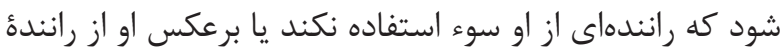

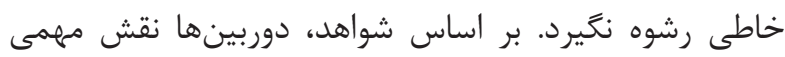

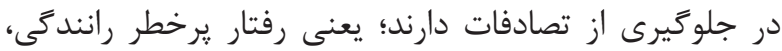

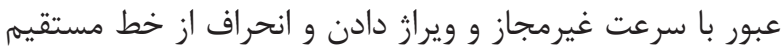

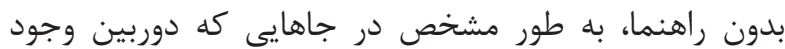

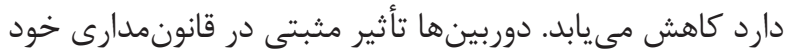

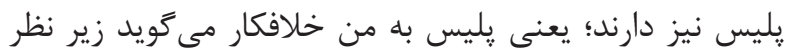

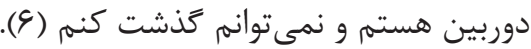

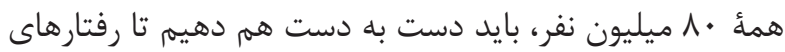

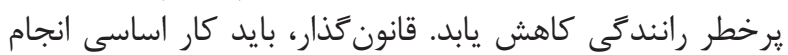

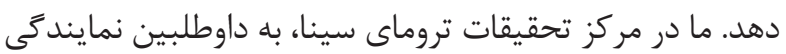

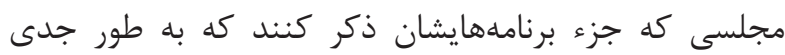

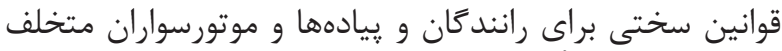

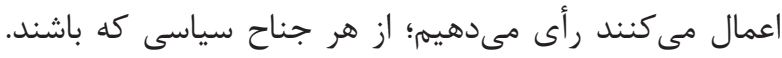

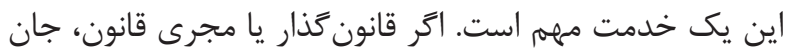

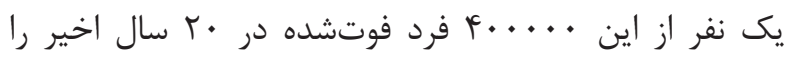

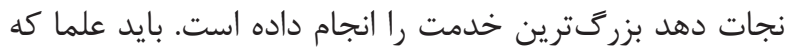

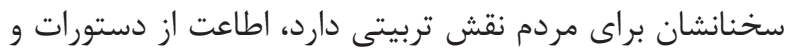

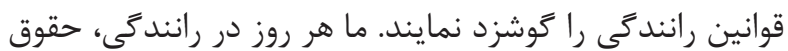

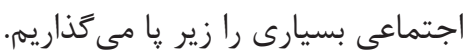

با تخلف و يارك دوبله كردن و راهبندان ايجاد كردن، وقت

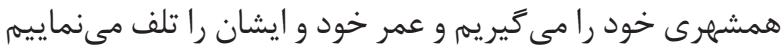

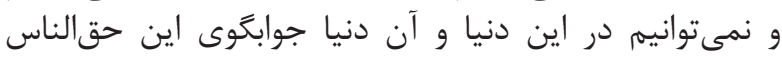

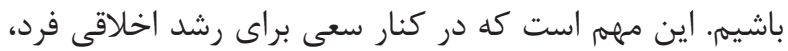

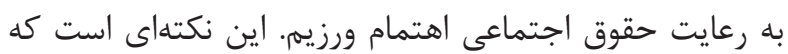

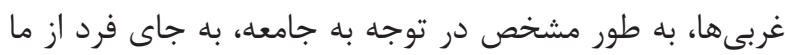

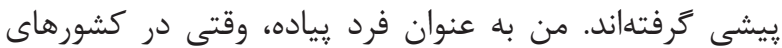

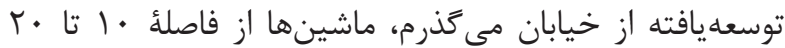


ايمن، وسيله نقليه ايمن و سيستم ايمن نياز داريم؛ يعنى ما به

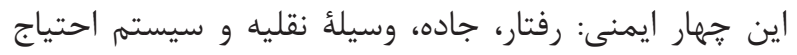

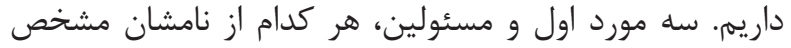

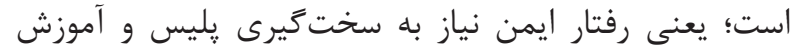

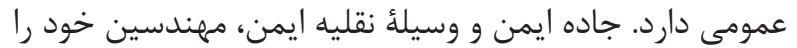

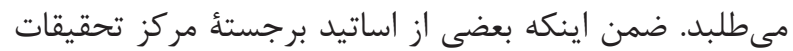

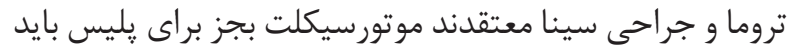

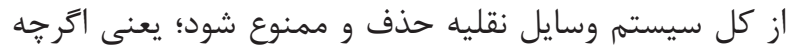

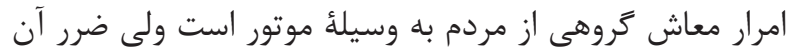

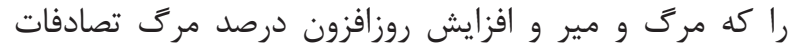

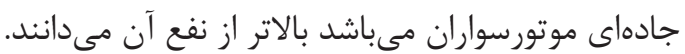

من به طور جدى معتقدم كه لازم است سخت گيرى بسيار شديد

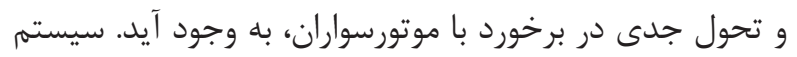

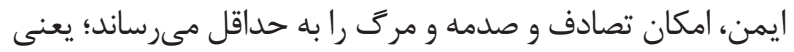

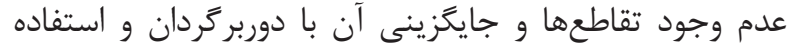

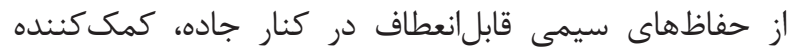

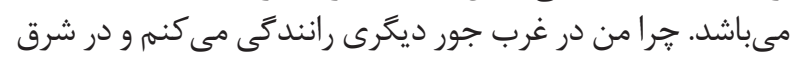

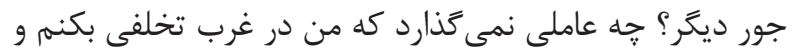

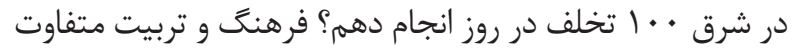

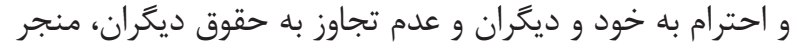

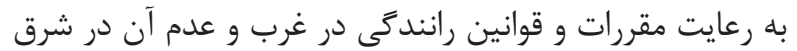

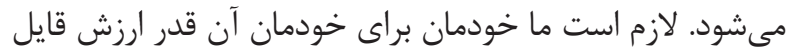

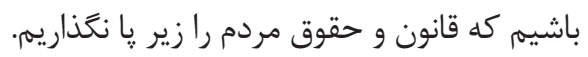

جهت موفقيت جدى در ييشخيرى، درمان، نوتوانى و ارزيابى در

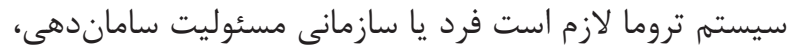

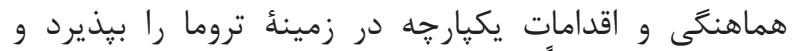

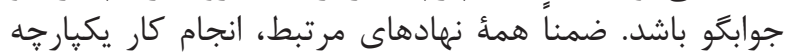

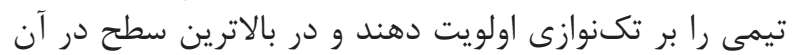
مشاركت نمايند.

1. Rasouli MR, Saadat S, Haddadi M, Gooya MM, Afsari M, Rahimi-Movaghar V. Epidemiology of injuries and poisonings in emergency departments in Iran. Public Health. 2011; 125(10): 727-33.

2. Rahimi-Movaghar V, Zarei MR, Saadat S, Rasouli MR, Nouri M. Road traffic crashes in Iran from 1997 to 2007. Int J Inj Contr Saf Promot. 2009; 16: 179-81.

3. Rasouli MR, Nouri M, Zarei MR, Saadat S, RahimiMovaghar V. Comparison of road traffic fatalities and injuries in Iran with other countries. Chin J Traumatol. 2008; 11: 131-4.

4. Shams M, Rahimi-Movaghar V. Risky driving behaviors in Tehran, Iran. Traffic Inj Prev. 2009; 10: 91-4.
مصدوم بيمارستانى مىباشد (| (1-1)). اين وضعيت در مورد

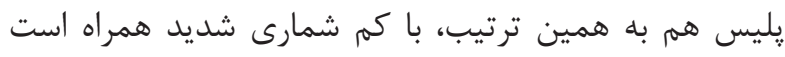

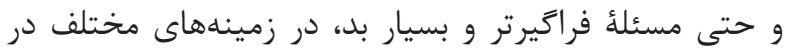

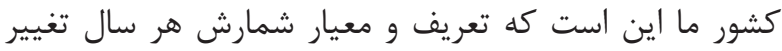

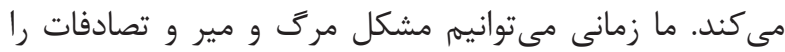

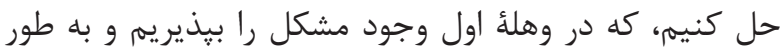

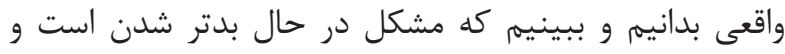

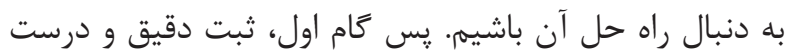

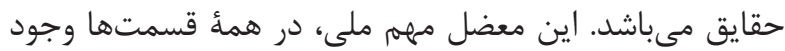

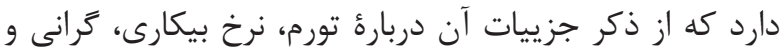

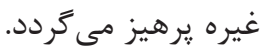

اكثر مردم مى دانند به هنگام موتورسوارى، بايد از كلاه ايمنى

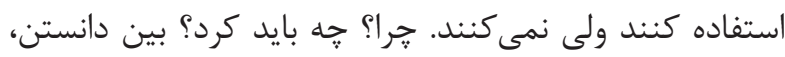

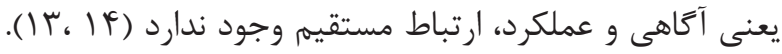

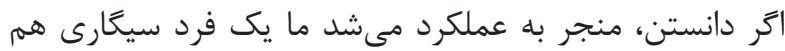

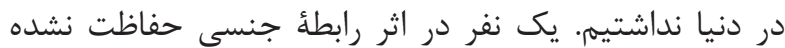

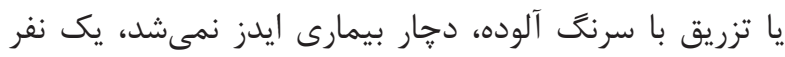

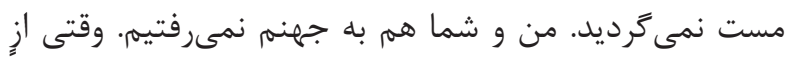

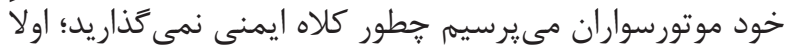

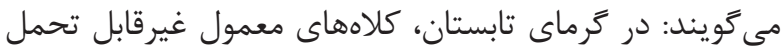

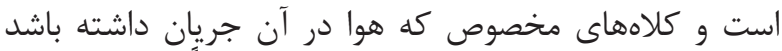

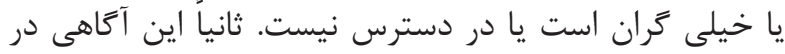

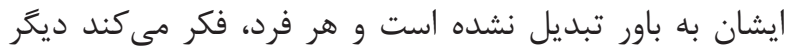

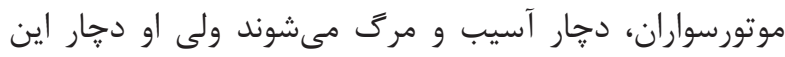

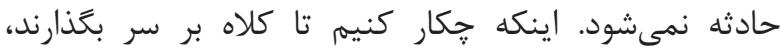

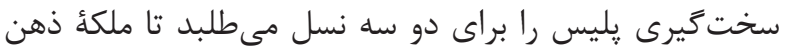

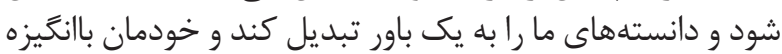

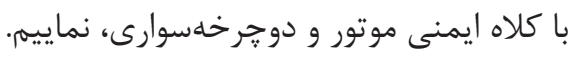

دربارهٔ عوامل اصلى كاهنده سوانح و تروما، به رفتار ايمن، جاده

منابع

5. Zargar M, Kalantar Motamedi SM, Karbakhsh M, Ghodsi SM, Rahimi-Movaghar V, Panahi F, et al. Trauma care system in Iran. Chin J Traumatol. 2011; 14: 131-6.

6. Rahimi-Movaghar V. Factors involved in the past and present history of road traffic injuries and deaths in Iran. Arch Iran Med. 2010; 13: 172-3.

7. Chardoli M, Rahimi-Movaghar V. Analysis of trauma outcome at a university hospital in Zahedan, Iran using the TRISS method. East Afr Med J. 2006; 83: 440-2.

8. Hafezi-Nejad N, Rahimi-Movaghar A, Motevalian A, Amin-Esmaeili M, Sharifi V, Hajebi A, et al. Populationbased incidence and cost of non-fatal injuries in Iran: a consistent under-recognized public health concern. Public Health. 2015; 129: 483-92. 


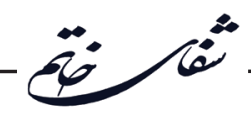

9. Salamati P, Rahimi-Movaghar A, Motevalian SA, Amin-Esmaeili M, Sharifi V, Hajebi A, et al. Incidence of self-reported interpersonal violence related physical injury in Iran. Iran Red Crescent Med J. 2015; 17: e15986. doi: 10.5812/ircmj.15986.

10. Hafezi-Nejad N, Rahimi-Movaghar A, Motevalian A, Amin-Esmaeili M, Sharifi V, Hajebi A, et al. A nationwide population-based study on incidence and cost of non-fatal injuries in Iran. Inj Prev. 2014 Oct;20(5):e9. doi: 10.1136/injuryprev-2013-041127.

11. Rahimi-Movaghar V. Controlled evaluation of injury in an international Safe Community: Kashmar, Iran. Public Health. 2010; 124: 190-7.
12. Motevalian SA, Haddadi M, Akbari H, Khorramirouz R, Saadat S, Tehrani A, et al. Strengthening injury surveillance system in Iran. Chin J Traumatol. 2011; 14: 348-53.

13. Mirzaei R, Hafezi-Nejad N, Sadegh Sabagh M, Ansari Moghaddam A, Eslami V, Rakhshani F, et al. Dominant role of drivers' attitude in prevention of road traffic crashes: a study on knowledge, attitude, and practice of drivers in Iran. Accid Anal Prev. 2014; 66: $36-42$.

14. Jafarpour S, Rahimi-Movaghar V. Determinants of risky driving behavior: a narrative review. Med J Islam Repub Iran. 2014; 28(1): 953-60. 